\title{
Optical Absorption in Overcoats of Nanoparticle Arrays on a Metallic Substrate
}

\author{
Vo-Van Truong', Bernard de Dormale ${ }^{2}$ \\ ${ }^{1}$ Department of Physics, Concordia University, 7141 Sherbrooke W, SP 367.03, Montréal, Québec, Canada \\ H4B 1R6 \\ ${ }^{2}$ Département de mathématiques et de statistique, Université de Moncton, Moncton, Nouveau-Brunswick, \\ Canada E1A 3E9
}

\begin{abstract}
Surface plasma oscillations in metallic particles as well as in thin metallic films have been studied extensively in the past decades. New features regarding surface plasma excitations are however constantly discovered, leading, for example, to surface-enhanced Raman scattering studies and enhanced optical transmission though metal films with nanohole arrays. In the present work, the role of a metallic substrate is examined in two cases, one involving an overcoat of dielectric nanoparticles and the other, an overcoat of metallic nanoparticles. Theoretical results are obtained by modeling the nanoparticles as forming a two-dimensional, hexagonal lattice of spheres. The scattered electromagnetic field is then calculated using a variant of the Green function method.

Comparison with experimental results is made for nanoparticles of tungsten oxide and tin oxide deposited on either gold or silver substrates, giving qualitative agreement on the extra absorption observed when the dielectric nanoparticles are added to the metallic surfaces. Such absorption would be attributed to the mirror-image effects between the particles and the substrate. On the other hand, calculations of the optical properties of silver or gold nanoparticle arrays on a gold or a silver substrate demonstrate very interesting features in the spectral region from $400 \mathrm{~nm}$ to $1000 \mathrm{~nm}$. Interactions between the nanoparticle arrays surface plasmons and their images in the metallic substrate would be responsible for the red shift observed in the absorption resonance. Moreover, effects of particle size and ambient index of refraction are studied, showing a great potential for applications in biosensing with structures consisting of metallic nanoparticle arrays on metallic substrates.
\end{abstract}

Key words: nanoparticles, plasmonics, optical properties, biosensing 


\section{Introduction}

Studies of the optical properties of metallic particles have been done extensively in the past [1-3], even long before the introduction of terms such as nanoparticles and plasmonics. Because of their peculiar absorption in the visible region of the spectrum not observed with bulk metals, metallic particles have attracted a great deal of attention from researchers who then realized that this absorption - attributed to surface plasma oscillations - could be exploited for various applications (optical filters [4], solar absorbers [5], sensors [6]...). With the improvements in methods for fabricating metallic particles at nanometric sizes and assembling them in various patterns, there has been a renewed interest because of the possibility of designing nanostructures that possess properties tailored to the application needs. Moreover, new features related to surface plasma excitations are constantly found, notably in surface-enhanced Raman scattering studies [7] and enhanced optical transmission through arrays of nanoholes in metallic films [8].

A frequently used approach to model films consisting of nanoparticles was the effectivemedium theory that approximated the granular films to an effective film having an effective optical thickness and effective optical properties [1-3]. The particles were considered very small compared to the light wavelength so that the dipole approximation could be used. This approach has served well the characterization of films in many studies, giving a good correlation between theory and experiments. When particles get slightly larger, the dipole approximation may no longer hold and retardation effects may also be taken into account.

In the present work, because of the interest that they may generate, the role of a metallic substrate is examined in two cases, one involving an overcoat of dielectric nanoparticle arrays and the other, an overcoat of metallic nanoparticles. The theoretical treatment of the scattered field is based on a variant of the electromagnetic Green function method for a two-dimensional lattice of spheres. The basis of the method is briefly outlined in section 2. A more comprehensive account would be far too technical and will be avoided. A detailed explanation of the approach can be found elsewhere [9].

\section{Outline of the method}

The problem at hand is the reflection/diffraction of a monochromatic electromagnetic field $\boldsymbol{E}^{\text {in }}, \boldsymbol{B}^{\text {in }}$ of wave vector $\boldsymbol{k}$ by a structure made of identical, non overlapping spheres of diameter $D$ lying on a substrate. The centers $\boldsymbol{r}_{n}=\left(x_{n}, y_{n}, 0\right)$ of these spheres are supposed to form a two-dimensional lattice in the $x y$-plane. For simplicity, we will assume that $\boldsymbol{r}_{0}=0$. We will call $\boldsymbol{E}_{n}, \boldsymbol{B}_{n}$ the field radiated by the sphere with center $\boldsymbol{r}_{n}$, and $\boldsymbol{E}_{n}^{\prime}, \boldsymbol{B}_{n}^{\prime}$ the total electromagnetic field incident on the same sphere. Because of the periodicity, we will have

$\boldsymbol{E}_{n}(\boldsymbol{r})=e^{i k r_{n}} \boldsymbol{E}_{0}\left(\boldsymbol{r}-\boldsymbol{r}_{n}\right), \boldsymbol{B}_{n}(\boldsymbol{r})=e^{i k \boldsymbol{r}_{n}} \boldsymbol{B}_{0}\left(\boldsymbol{r}-\boldsymbol{r}_{n}\right)$

and similarly for $\boldsymbol{E}_{n}^{\prime}, \boldsymbol{B}_{n}^{\prime}$. Therefore, only $\boldsymbol{E}_{0}, \boldsymbol{B}_{0}$ and $\boldsymbol{E}_{0}^{\prime}, \boldsymbol{B}_{0}^{\prime}$. need to be known.

The principle of the method consists in using the multipole moments $a_{E}(l, m), a_{M}(l, m)$ of $\boldsymbol{E}_{0}, \boldsymbol{B}_{0}$ to compute the field radiated by the whole structure. To obtain them, one needs to establish a set of equations that relate them to the multipole moments $a_{E}^{\prime}(l, m), a_{M}^{\prime}(l, m)$ of $\boldsymbol{E}_{0}^{\prime}, \boldsymbol{B}_{0}^{\prime}$ and those of the incoming field, $a_{E}^{i n}(l, m), a_{M}^{i n}(l, m)$. To this effect, one would be tempted to write

$$
\boldsymbol{E}_{0}^{\prime}=\boldsymbol{E}^{i n}+\sum_{n \neq 0} \boldsymbol{E}_{n}+\boldsymbol{E}^{r e}, \boldsymbol{B}_{0}^{\prime}=\boldsymbol{B}^{i n}+\sum_{n \neq 0} \boldsymbol{B}_{n}+\boldsymbol{B}^{r e}
$$


where $\boldsymbol{E}^{r e}, \boldsymbol{B}^{r e}$ is the field reflected by the substrate, together with (1). This is actually the starting point of many approaches [10-12]. Numerical exploration, however, reveals that the series in (2) do not converge, due to the long range nature of the radiation field [10]. It is thus advisable to take another avenue.

A better approach is provided by Ewald's method [13]. It more or less amounts to obtaining $\boldsymbol{E}_{0}^{\prime}, \boldsymbol{B}_{0}^{\prime}$ by computing the field radiated by the whole layer of spheres, using Fourier series, then subtracting the contribution of the sphere with center $\boldsymbol{r}_{0}$. Once the sought relation between $a_{E}(l, m), a_{M}(l, m), a_{E}^{\prime}(l, m), a_{M}^{\prime}(l, m)$ and $a_{E}^{i n}(l, m), a_{M}^{i n}(l, m)$ is established, a closure to the problem is brought by Mie's theory[14, 15], which states that one must have

$a_{E}(l, m)=\alpha_{E}(l) a_{E}^{\prime}(l, m), a_{M}(l, m)=\alpha_{M}(l) a_{M}^{\prime}(l, m)$

where $\alpha_{E}(l)$ and $\alpha_{M}(l)$ are known functions of the composition and size of the spheres.

What mainly sets this approach apart from other ones is that, by using the Fourier formalism from the very start, we completely avoid having to deal with ill-defined quantities. As an added benefit, since the radiated field is now expressed as a superposition of plane and vanishing waves, it is now quite easy to take into account its reflection by the substrate.

\section{Dielectric nanoparticles on metallic surfaces}

It has been shown that overcoats of dielectric nanoparticles on gold or silver surfaces can provide a mechanism for coupling with the incident light for the excitation of surface plasmons via mirror-image effects [16-18]. In the previous works, experimental features observed could be qualitatively accounted for by an effective-medium approach approximating the particle to a dipole interacting with its mirror-image in the metallic substrate and the surrounding dipoles and their mirror-images, while neglecting retardation effects. Experimental evidence of a resonant absorption was provided by measuring the reflectance of metallic substrates overcoated with a granular transparent film that showed a drop in its value near the resonant wavelength. When the overcoat is very thin, this drop has a shoulder-like structure that is not very well defined [18]. This structure can be better seen in the normalized differential reflectance given by $\Delta R / R$ where $\Delta \mathrm{R}$ is the difference between the reflectance of the bare metallic surface and that of the overcoated metallic surface, and $\mathrm{R}$ is the reflectance of the bare metallic surface. The use of differential reflection spectroscopy for studying very thin adsorbed films on a reflecting substrate has been well described in the literature [19-20]. Because of its high sensitivity to small changes on surfaces, it is preferred over simple reflectance measurements. It should also be mentioned that in all the following, both calculations and experimental measurements are for unpolarized incoming light at normal incidence.

In the present work, instead of this effective-medium approach, calculations are made using the theoretical treatment of the scattered field outlined at Sect. 2 and the supposition of an hexagonal array of nanoparticles of spherical shape characterized by the diameter $D$ of the sphere and the distance $d$ separating two adjacent spheres (interparticle distance). The parameters $D$ and $d$ are chosen in such a way to reflect experimental situations.

Fig. 1 shows the calculations performed for the differential reflectance in the case of a gold surface overcoated with a hexagonal array of tin dioxide nanoparticles. The particle diameter is $20 \mathrm{~nm}$ and the inter-particle distance respectively equal to 25,30 and $40 \mathrm{~nm}$. Optical constants are provided by $[21,22]$. An absorption structure can be found near the wavelength of $475 \mathrm{~nm}$, a location near the one observed in experimental measurements (at $490 \mathrm{~nm}$ ) reported elsewhere [23] with tin oxide nanoparticles synthesized by a sol-gel 
method and deposited on a gold surface by a dip coating process. It can be seen that the overall shape of the calculated curves agrees well with experiments (Fig. 2). It should be noted that the location of this absorption structure is dependent upon the particle shape and an oblate spheroid on the film plane, for example, will make the absorption wavelength shifted to larger values (red shift) and contribute to the difference found between theory and experiment. These spheroidal particles have effective depolarization factors that depend on different factors that also include the particle axial ratio. Within the dipole approximation and taking into account mirror-image effects in the substrate, the expression derived for the differential reflectance of an effective layer consisting of these spheroidal nanoparticles indeed showed a dependence of the absorption structure on the particle shape [18]. Such a phenomenon would result from the interaction between the dielectric particles and the substrate via mirror-image effects. A phenomenological study of the effect of a transition layer on the dispersion of surface polaritons in a metallic substrate had been made by Agranovich et al. [24] and could serve as a basis for a more throughout interpretation of the absorption observed.

In Fig. 3, a second sample calculation is performed. In this case, the differential reflectance for tungsten trioxide (a high refractive index dielectric material, with n near 2 in the visible region) nanoparticle arrays on silver surface is presented with $D=20 \mathrm{~nm}$ and $d=25,30,40 \mathrm{~nm}$. The optical constants for silver and tungsten trioxide are provided by [21]. Two resonant absorption structures can be found in this case, one near $345 \mathrm{~nm}$ and another at $315 \mathrm{~nm}$. The structure at $345 \mathrm{~nm}$ (for spherical particles) would be attributed to the resonant absorption observed experimentally at longer wavelengths with "flatter" tungsten trioxide particles in [17] in the region around $400 \mathrm{~nm}$ (Fig. 4). At 315 $\mathrm{nm}$ or near of it, there are the bulk plasma absorption in silver, normally found at $327 \mathrm{~nm}$, as well as interband transitions [25], as demonstrated by the high peak observed in the differential reflectance.

Basically, for dielectric nanoparticles deposited on a metallic surface, the resonant absorption structure that was found in previous experiments [16-18] can also be found in our calculations based on the scattered field theoretical model for a lattice of spherical particles, a more rigorous approach than the effective-medium approximation. As observed previously, this absorption occurs at a wavelength near the surface plasma wavelength of the metal used as the substrate. Furthermore, when the index of the dielectric particle is varied, it was observed that the larger the refractive index of the nanoparticle deposit, the more important is the absorption phenomenon. Comparison effectively has been made between $\mathrm{WO}_{3}$ (refractive index near 2) and $\mathrm{MgF}_{2}$ (refractive index $=1.38$ ) particle deposits on $\mathrm{Ag}$ surfaces and structures found in the differential reflectance for $\mathrm{WO}_{3}$ were much more pronounced than those for $\mathrm{MgF}_{2}[17,18]$.

\section{Metallic particles on metallic surfaces}

With the ease in fabricating metallic particles and different methods for depositing them on metallic surfaces, it would be interesting to examine the properties of such a combination, especially when it is expected that plasmonic properties of the deposits can be altered or influenced by the properties of the substrate. For our calculations a gold substrate is used and the nanoparticles are either gold or silver spheres with nanometric sizes. The substrate is considered to be a semi-infinite medium and therefore a dip in the reflectance curves for the system consisting of nanoparticles on the metallic surface will locate the absorption wavelength for such a system. In the present section, reflectance (R) data are presented because the absorption features found here are prominent and there is no need to use a higher sensitivity method such as the differential reflectance spectroscopy in the previous section. As the substrate is semi-infinite, absorption in this case can be depicted in a peak position in $A=1-R$ or a dip in $R$.

Fig. 5 shows the results of calculations performed for $10 \mathrm{~nm}$ Au spheres in a hexagonal array with an inter-particle distance $d=10,11,12,13,15 \mathrm{~nm}$. When the particles are well 
separated from each other $(d=15 \mathrm{~nm})$, the reflectance curve resembles more to the one for the substrate alone, i.e. the Au surface. This is expected since when particles are well separated from each other, their filling factor is very small and their influence on the overall optical properties minimized. When the particles get closer and closer, there is a structure appearing in the curve that becomes more and more pronounced, shifting to longer wavelengths. With $d=10 \mathrm{~nm}$, a well-defined minimum can be seen near $662 \mathrm{~nm}$. Such an absorption wavelength can be compared to the one for a similar hexagonal array of spheres in vacuum that occurs at about $628 \mathrm{~nm}$. It may not be entirely correct to suppose the inter-particle distance to be equal to the particle diameter but this case should be treated as the situation where the particles are very close to each other.

The plasma resonance in the Au particles is thus observed to be shifted to longer wavelengths due to interaction with the Au surface. Another effect is the sharpening of this resonant structure, making it better defined than in the case of a film of particles surrounded by vacuum. This latter conclusion is based on a comparison with detailed calculations in vacuum not shown here.

Similar results are obtained for Ag nanoparticles on a Au surface (Fig. 6). In this case, for $d=10 \mathrm{~nm}$, the dip in the reflectance of the nanoparticle arrays on Au occurs near $595 \mathrm{~nm}$ whereas the absorption for a nanoparticle film in vacuum is found at $522 \mathrm{~nm}$. Similarly to $\mathrm{Au}$ particles on $\mathrm{Au}$, the shift of the resonant wavelength to longer wavelengths is also accompanied by a sharpening of the absorption structure.

In increasing the nanoparticle diameter to $20 \mathrm{~nm}$, a further shift to longer wavelengths can be observed (Fig. 7). In this latter case, for the particles very close to each other $(d=$ $20 \mathrm{~nm}$ ), the resonant absorption occurs near $660 \mathrm{~nm}$. Such a location of the resonance wavelength together with the appreciable shift observed in varying the inter-particle distance would make such a structure very interesting for applications involving for example strains and stresses in the substrate.

Realizing the potential for such systems in detecting bio-species, calculations have been made by varying $\mathrm{n}$, the incident ambient medium refractive index, from 1.00 to 1.42 for both $\mathrm{Ag}$ and $\mathrm{Au}$ spheres on $\mathrm{Au}$ surfaces. Such variations of the refractive index are representative of a good range of bio-species that are to be detected.

If $\mathrm{n}$ changes from 1.33 (refraction index for water) to 1.42 , the shift observed in the case of $10 \mathrm{~nm} \mathrm{Ag} \mathrm{nanoparticles} \mathrm{on} \mathrm{a} \mathrm{Au} \mathrm{surface} \mathrm{is} \mathrm{from} 750 \mathrm{~nm}$ to $800 \mathrm{~nm}$, giving a large detection sensitivity of $556 \mathrm{~nm} / \mathrm{RIU}$ (refractive index unit) (Fig.8). This can be compared favourably to a sensitivity of $439 \mathrm{~nm} / \mathrm{RIU}$ for similar Ag nanoparticles in vacuum. When a system consisting of $10 \mathrm{~nm} \mathrm{Au}$ nanoparticles on $\mathrm{Au}$ is considered, the shift for a similar range of variation for $\mathrm{n}$ is from 786 to $827 \mathrm{~nm}$ (Fig. 9), resulting in a sensitivity of 455 $\mathrm{nm} / \mathrm{RIU}$ that is higher than $412 \mathrm{~nm} / \mathrm{RIU}$ found for $\mathrm{Au}$ nanoparticles in vacuum. Such large values of the sensitivity found with $\mathrm{Ag}$ and $\mathrm{Au}$ particles on $\mathrm{Au}$ surfaces can certainly be exploited in the designing of biosensors.

\section{Conclusion}

We have shown in the present work that nanoparticles, whether dielectric or metallic, when deposited on a metallic surface, present very interesting absorption features that would be associated to surface plasmon phenomena. The scattered field approach used was able to take into account retardation effects as well as multipole contributions to the optical properties of the nanostructured systems considered. Calculations based on this approach have confirmed results found in previous experiments with dielectric tin dioxide and tungsten trioxide particles. As these properties can be significantly affected by the ambient environment, the use of such systems as biosensors should be considered, 
especially when detection sensitivity can be as high as $550 \mathrm{~nm} / \mathrm{RIU}$, as observed in the case of 10nm Ag nanoparticles on a Au surface.

\section{Acknowledgements}

The financial support of the Natural Sciences and Engineering Research Council (NSERC) is gratefully acknowledged. We also thank Professor Georges Bader for kindly providing us with the optical constants for $\mathrm{Au}, \mathrm{Ag}$ and $\mathrm{WO}_{3}$. 


\section{References}

1. T. Yamaguchi, S. Yoshida, A. Kinbara, Thin Solid Films 21, 173 (1974).

2. S. Norrman, T. Anderson, C.G.Granqvist, O. Hundersi, Phys. Rev. B 18, 674 (1978).

3. V.V. Truong and G.D.Scott, J. Opt. Soc. Am. 67, 502-510 (1977).

4. S. Nonaka, T. Suda, H. Oda, Jpn. J. Appl. Phys. 41, 4538 (2002).

5. C.G. Granqvist and O. Hunderi, Appl. Phys. Lett. 32, 798 (1978).

6. S. Enoch, R. Quidant, G. Badenes, Optics Express 12, 3422 (2004).

7. J.D. Driskell, R.J. Lipert, M.D. Porter, J. Phys. Chem. B 110, 17444 (2006).

8. C. Genet and T.W. Ebbesen, Nature 445, 39 (2007).

9. B.M. de Dormale and V.V. Truong, Diffraction of light by a two-dimensional lattice of spheres, preprint, Université de Moncton (2009); B.M. de Dormale, Can. J. Phys. 74, 43 (1996); B.M. de Dormale, Can. J. Phys. 74, 603 (1996); B.M. de Dormale, Can. J. Phys. 74, 608 (1996).

10. G. Bosi, J. Opt. Soc. Am. B 11, 1073 (1994).

11. N. Stefanou and A. Modinos, J. Phys.: Condens. Matter 3, 8135 (1991).

12. D. Bedeaux and J. Vlieger, Optical properties of surfaces, Imperial College Press, London (2002)

13. J.M. Ziman, Principles of the theory of solids, $2^{\text {nd }}$ ed., Cambridge University Press, Cambdidge (1972).

14. J.D. Jackson. Classical electrodynamics, $2^{\text {nd }}$ ed., John Wiley and sons, New York (1975).

15. M. Born and E. Wolf, Principles of optics, 6th ed., Pergamon Press, Oxford (1980).

16. V.V. Truong and T. Yamaguchi, Bull. Res. Inst. Electron. (Japan) 25, 1 (1990).

17. V.V. Truong, P.V. Ashrit, G. Bader, P. Courteau, F.E. Girouard, T. Yamaguchi, Can. J. Phys. 69, 107 (1991).

18. P.V. Ashrit, G. Bader, F.E. Girouard, T. Richard, V.V. Truong, J. Appl. Phys. 70, 3797 (1991).

19. J.D.E. McIntyre, D.E. Aspnes, Surf. Sci. 24, 417 (1971).

20. Y. Borensztein, Phys. Stat. Sol. (a) 202, 1313 (2005).

21. G. Bader, $\mathrm{Au}, \mathrm{Ag}$ and $\mathrm{WO}_{3}$ Optical Constants, Université de Moncton, private communication (2008).

22. D. Davazoglou, Thin Solid Films 302, 204 (1997).

23. N. Seirafianpour, S. Badilescu, Y. Djaoued, R. Bruning, S. Balaji, M. Kahrizi, V.V. Truong, E-MRS 2008 paper (2008).

24. V.M. Agranovich, S.A. Darmanyan, A.G. Mal'chukov, Opt. Comm. 33, 234 (1980).

25. W. Steinmann, Phys. Stat. Sol. 28, 437 (1968). 


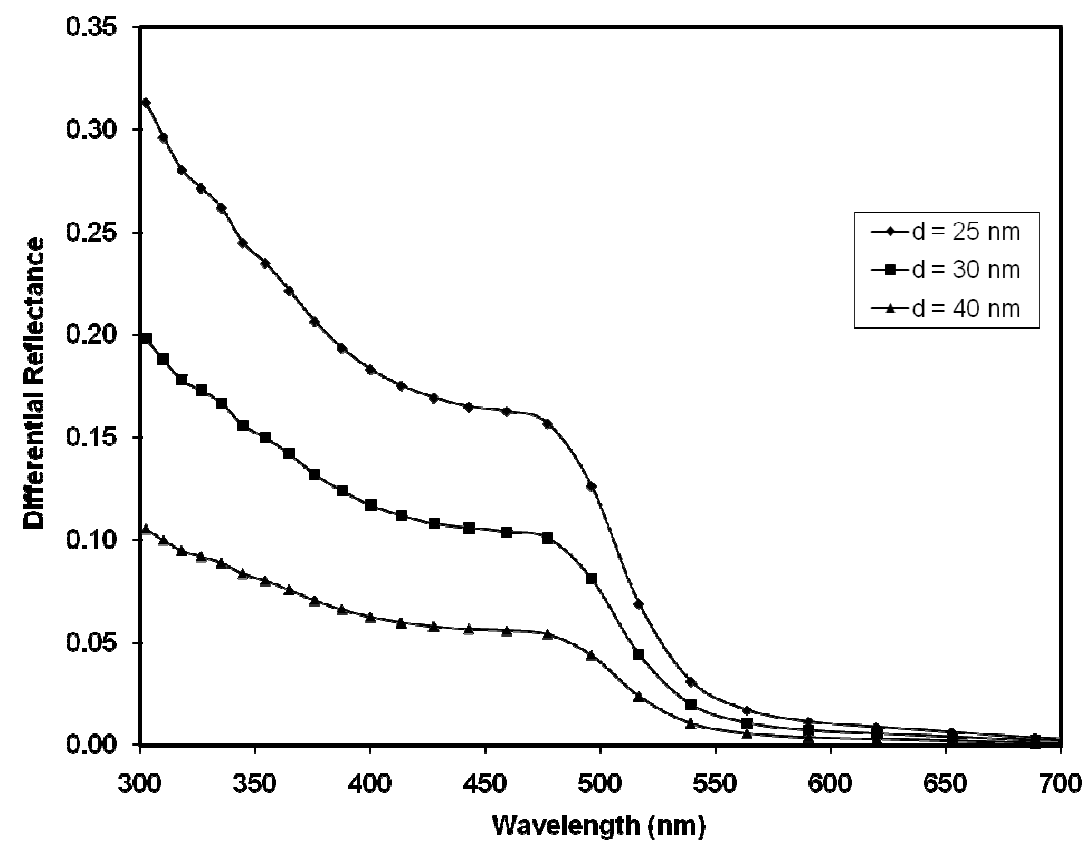

Figure 1 Calculated differential reflectance for $20 \mathrm{~nm} \mathrm{SnO}{ }_{2}$ spheres on $\mathrm{Au}$ with inter-particle distance $d=25,30,40 \mathrm{~nm}$.

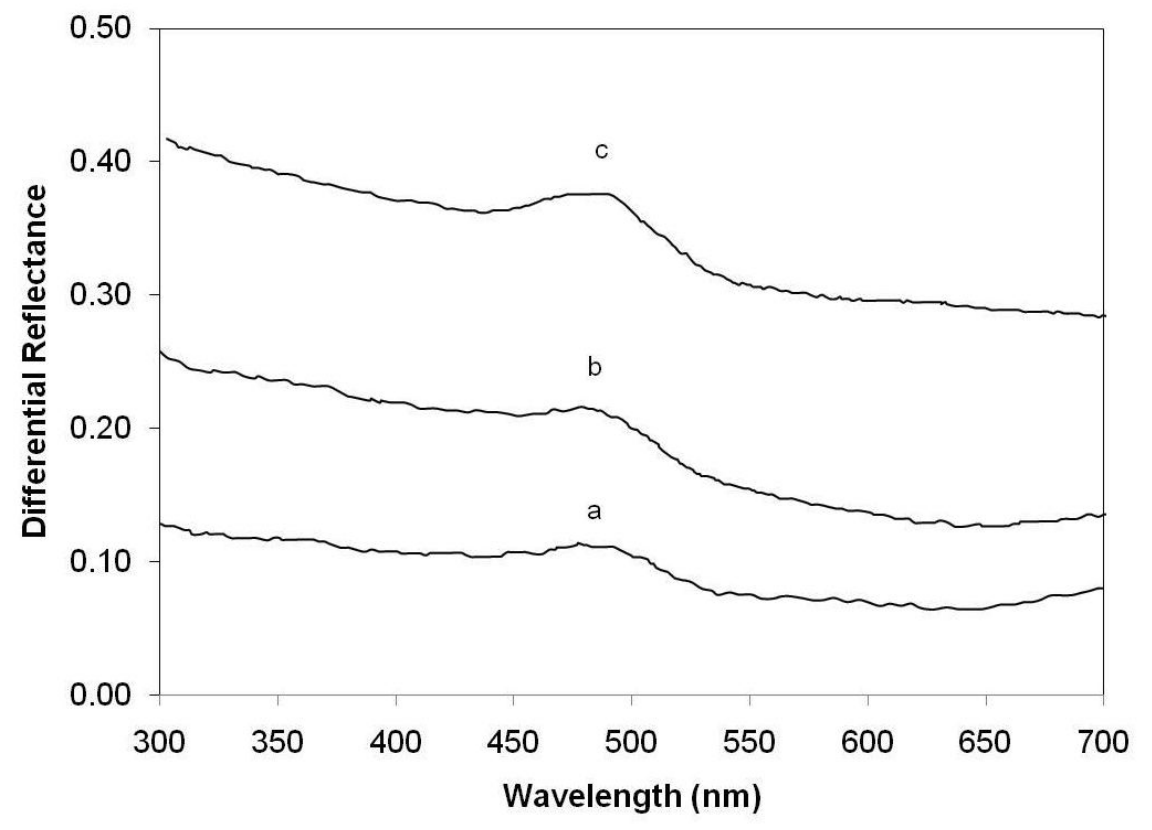

Figure 2 Experimental differential reflectance for $\mathrm{SnO}_{2}$ particles on $\mathrm{Au}$ surfaces prepared by a dip coating method. The $\mathrm{SnO}_{2}$ film thickness for a, b and c was 28,35 and $40 \mathrm{~nm}$, respectively. 


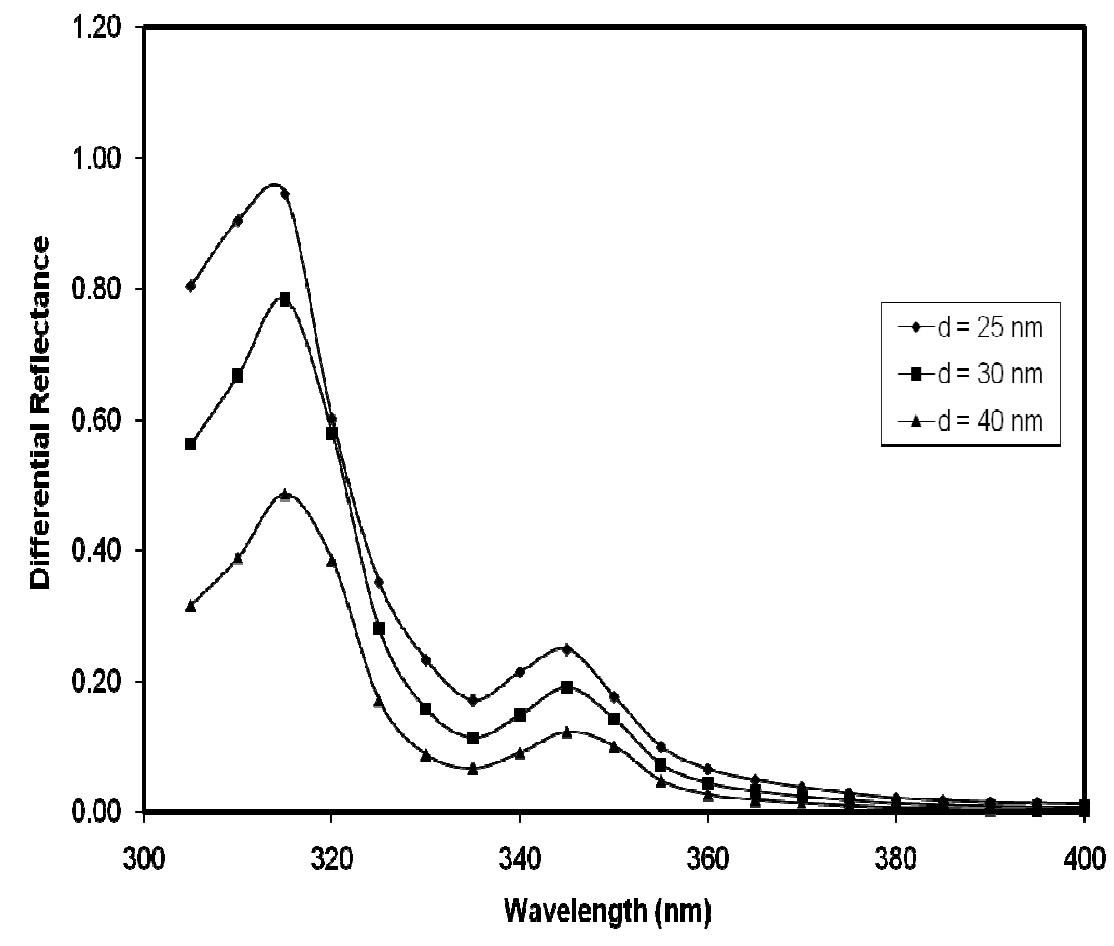

Figure 3 Calculated differential reflectance for $20 \mathrm{~nm} \mathrm{WO}_{3}$ spheres on $\mathrm{Ag}$ with inter-particle distance $\mathrm{d}=25,30,40 \mathrm{~nm}$.

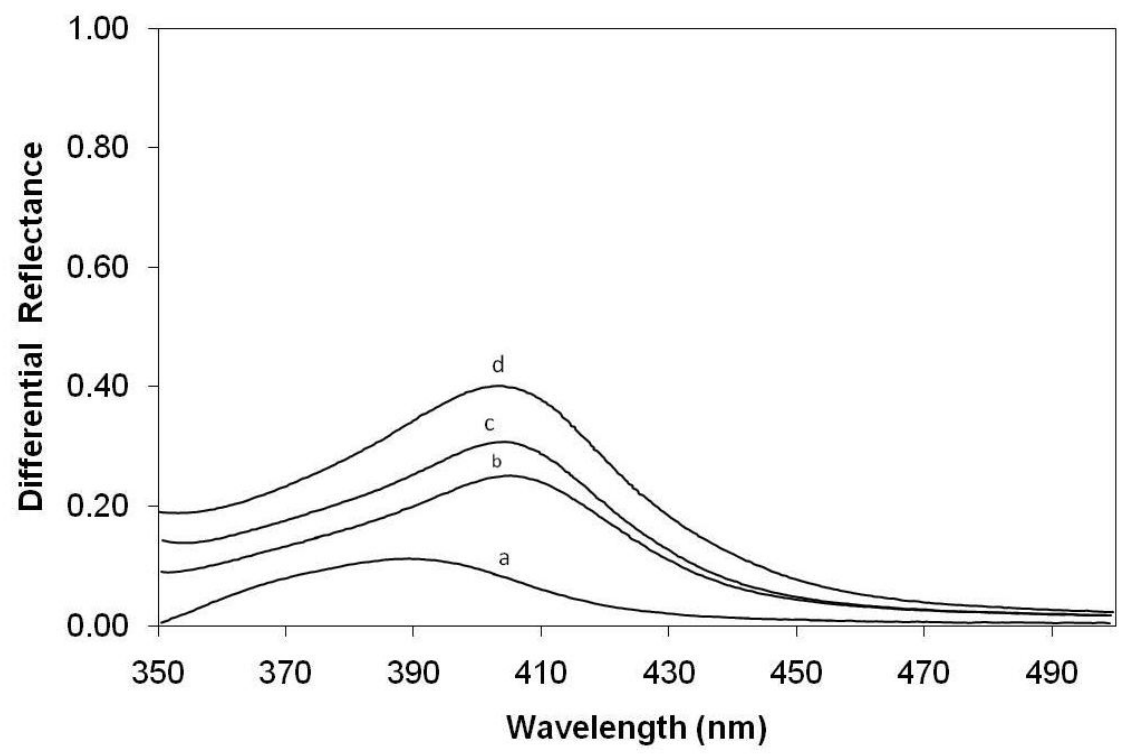

Figure 4 Experimental differential reflectance for $\mathrm{WO}_{3}$ particles on $\mathrm{Ag}$ prepared by thermal evaporation. The $\mathrm{WO}_{3}$ film thickness for $\mathrm{a}, \mathrm{b}, \mathrm{c}$ and $\mathrm{d}$ was $1.5,2.5,5.0$ and $7.0 \mathrm{~nm}$, respectively. 


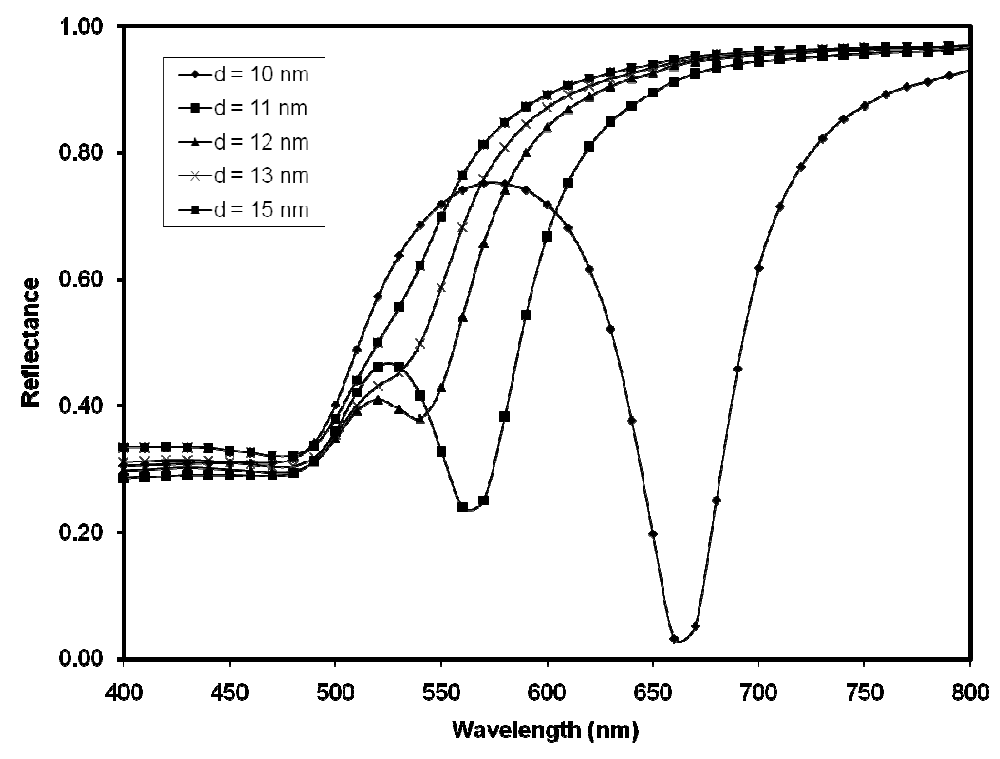

Figure 5 Calculated reflectance for $10 \mathrm{~nm}$ Au spheres on Au with inter-particle distance $d=10$, $11,12,13,15 \mathrm{~nm}$.

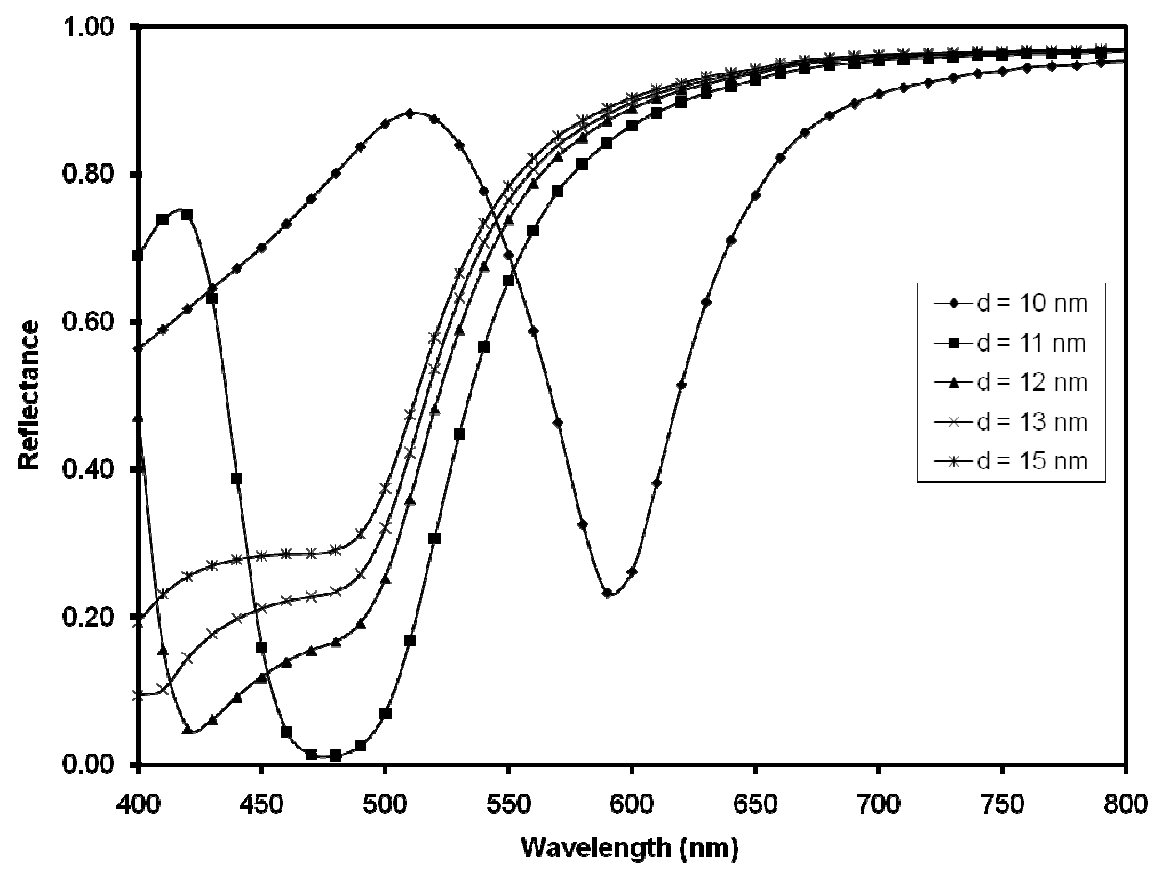

Figure 6 Calculated reflectance for $10 \mathrm{~nm} \mathrm{Ag} \mathrm{spheres} \mathrm{on} \mathrm{Au} \mathrm{with} \mathrm{inter-particle} \mathrm{distance} d=10$, $11,12,13,15 \mathrm{~nm}$. 


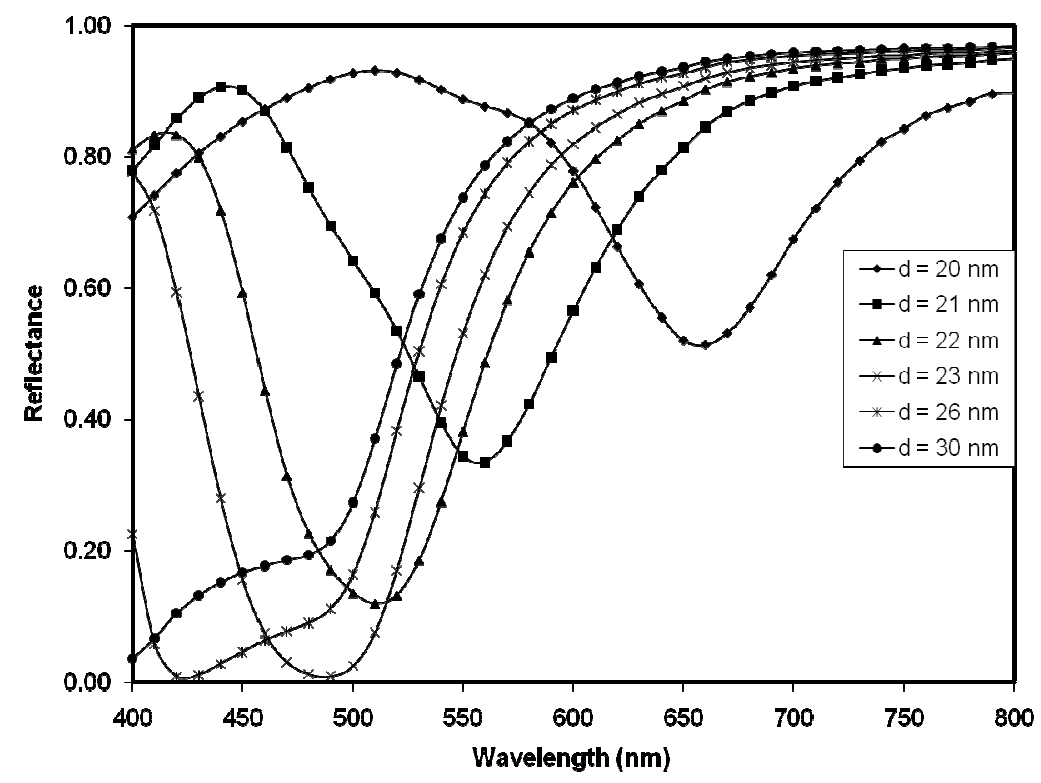

Figure 7 Calculated reflectance for $20 \mathrm{~nm} \mathrm{Ag} \mathrm{spheres} \mathrm{on} \mathrm{Au} \mathrm{with} \mathrm{inter-particle} \mathrm{distance} d=20$, $21,22,23,26,30 \mathrm{~nm}$.

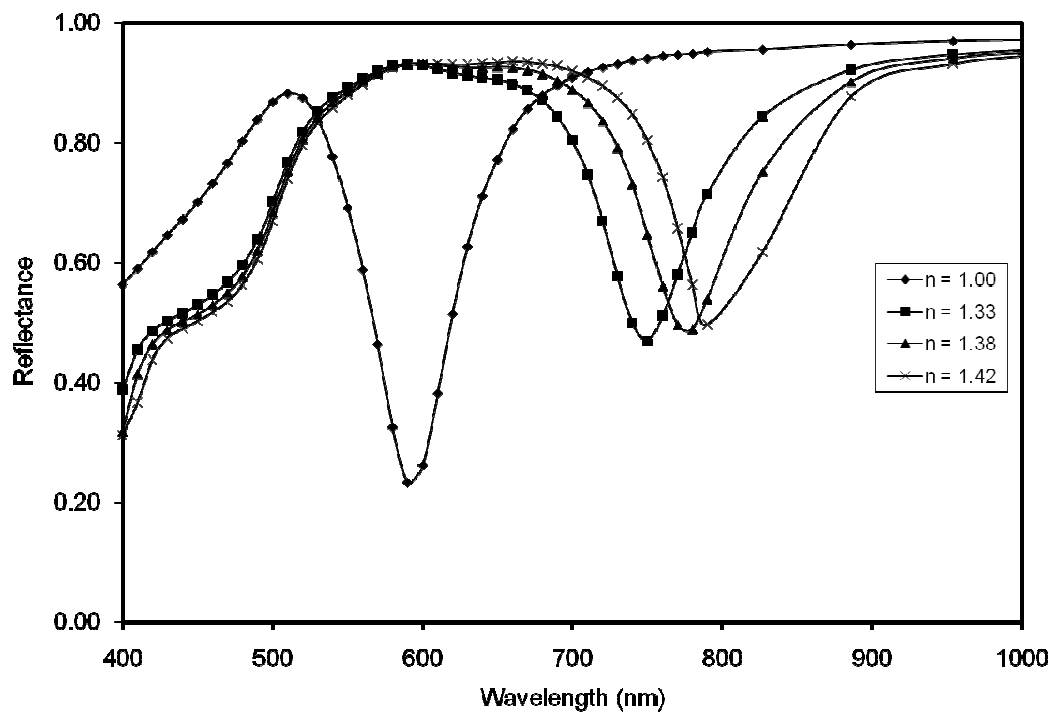

Figure 8 Calculated reflectance for $10 \mathrm{~nm} \mathrm{Ag}$ spheres on Au with inter-particle distance $d=10$ $\mathrm{nm}$ and incident ambient refractive index $n=1.00,1.33,1.38,1.42$. 


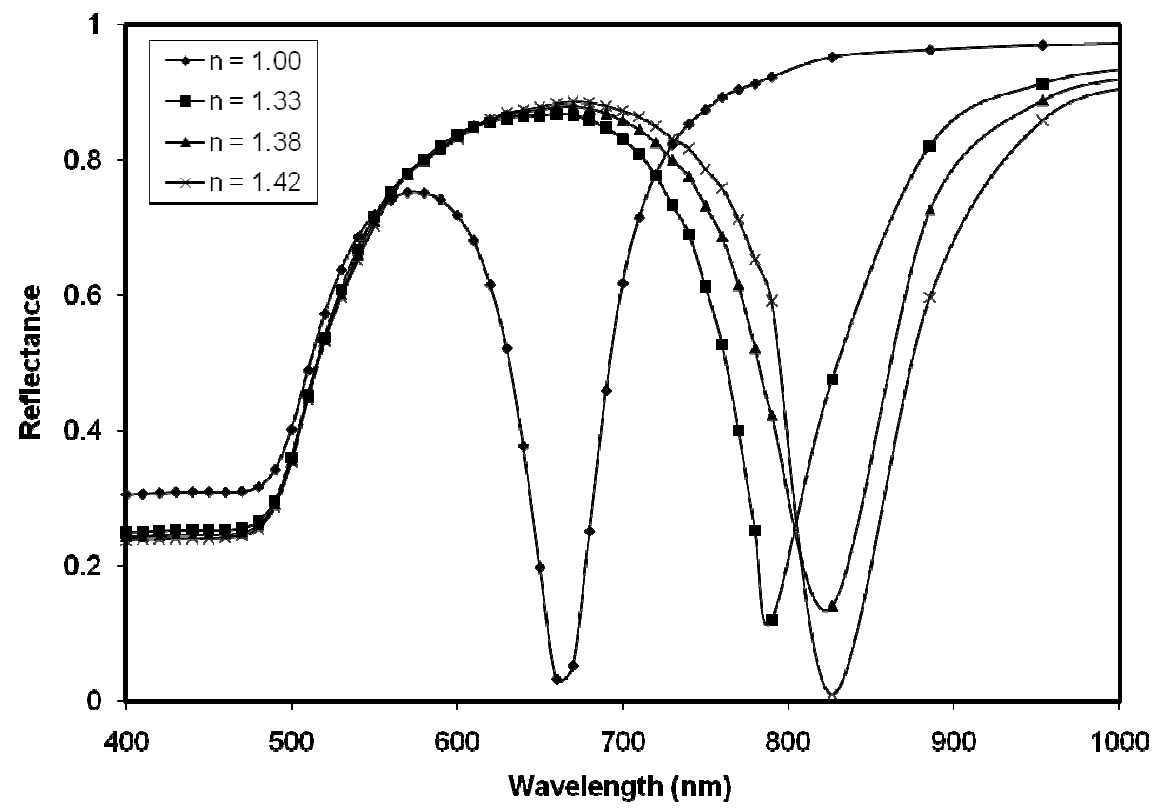

Figure 9 Calculated reflectance for $10 \mathrm{~nm}$ Au spheres on Au with inter-particle distance $\mathrm{d}=10$ $\mathrm{nm}$ and incident ambient refractive index $\mathrm{n}=1.00,1.33,1.38,1.42$. 